\title{
Pauses of larval development and its consequences for stage-structured populations
}

\author{
Hermann Brunner ${ }^{a}$, Stephen A. Gourley ${ }^{b},{\text { Rongsong } \text { Liu }^{c} \text { and Yanyu Xiao }}^{d}$ \\ ${ }^{a}$ Department of Mathematics, Hong Kong Baptist University, Hong Kong SAR, China and \\ Department of Mathematics and Statistics, Memorial University of Newfoundland, St. \\ John's, NL, Canada, A1C 5S7. \\ ${ }^{b}$ Department of Mathematics, University of Surrey, \\ Guildford GU2 7XH, UK. \\ ${ }^{c}$ Department of Mathematics and Department of Zoology and Physiology, University of \\ Wyoming, Laramie, WY, USA, 82071. \\ ${ }^{d}$ Department of Mathematical Sciences, McMicken College of Arts \& Sciences, University \\ of Cincinnati, Cincinnati, OH 45221-0025, USA.
}

\begin{abstract}
This paper considers some novel predictions of a mathematical model for a stagestructured insect species that undergoes diapause if faced with strong intra-specific competition among larvae. The model consists of a system of two delay differential equations with a state-dependent time delay of threshold type. When the model has an Allee effect, we show that diapause may cause extinction in some parameter regimes even where the initial population is high. We also demonstrate that the model can have diapause-induced periodic solutions that can arise even if the birth function is strictly increasing, a situation in which solutions for the constant delay case always converge to an equilibrium.
\end{abstract}

Short title: Novel phenomena in models of diapause

Keywords: delay; threshold condition; size-structure; diapause; Allee effect

AMS Classifications: 34K11, 34K13, 34K25, 92D40.

\section{Introduction}

Larval insects sometimes experience halting or slowing down of development, known as diapause. This is often in response to adverse environmental conditions such as drought or unfavourable temperatures, but it can also be a response to reduced food availability which, in turn, might be because of seasonal factors or as a consequence of intra-specific competition among the larvae at higher densities. In insects, diapause appears to be an adaptation enabling them to persist in regions that present a challenge to permanent habitation, or to maintain higher numbers than would otherwise be expected. Diapause can also induce a 
rhythm to the life cycle to synchronise with the rhythm of the environment, to ensure that active stages of the life cycle happen at times when food is abundant and other aspects of the environment favour survival (Andrewartha [1]).

There are numerous examples in nature of species in which competition may be a factor that can work to induce diapause in the larvae. Parasites sometimes arrest the development of younger stages as they await free space, to avoid competition (Combes [4]). GonzálezCandelas et al [7], in a study of larval competition in Drosophila Melanogaster, suggest that strong competition can halt development of the individuals until a time of increased food availability. Competition for food, among other factors such as low temperatures, desiccation and lack of aeration, can induce diapause in the larvae of various Lucilia species (Ring [19], and references therein). Madder et al [14] present the results of a number of experiments on the induction of diapause in Culex pipiens and $C$. Restuans. In one experiment, larvae were fed a limited daily amount of food for which they competed. This influenced their development at higher densities. The incidence of diapause was found to be reduced at lower larval densities although the effect of density on induction of diapause was temperature dependent.

In this paper we present a novel mathematical model for diapause in an insect species for which competition among the larvae for food may initiate diapause. We present the particularly novel finding that, far from enhancing survival prospects, in certain extreme parameter regimes it may result in the extinction of the species. Later we discuss the implications of this prediction by stressing the presence in nature of the adaptation of premature pupation, whereby in practice the species avoids extinction by cutting short larval development under conditions of food shortage, even though this results in smaller adults that are less fecund.

We assume that the species has two life stages, larva and adult, and we work with variables $I(t)$ and $A(t)$ that denote respectively the numbers at time $t$ of immature (larval) and adult insects. Our model consists of a system of two delay differential equations that involve a time delay $\tau(t)$ determined from a threshold condition. The model is formally derived in Gourley, Liu and Lou [8] and will be treated in this paper in a situation excluded from consideration in [8]: that in which larval development may pause completely (diapause). In [8], results were presented on boundedness and stability of equilibria, whereas in the present paper we focus on completely different issues raised by the possibility of diapause. These are: complete extinction of the population and the possibility of periodic solutions, both induced by diapause.

The previous paper [8] emphasized the importance of the fact that, in insects, metamorphoses is typically triggered when the larva has reached a certain size rather than a certain age (Callier and Nijhout [3], Blakley [2], Rewitz et al [18]). In Drosophila a large pulse of a steroid hormone known as 20-hydroxyecdysone (ecdysone) appears to be the trigger for pupariation, and it happens on the attainment of sufficient larval growth (Moeller et al [15], Rewitz et al [18]). If larvae grow at a steady rate that is always the same, independent of densities, environmental and seasonal factors, then the time taken for development from egg to maturation will be predictable and not vary much. Well studied stage-structured models with constant maturation delay, derived from age structured models using the McKendrickvon Foerster equation, can work well in such situations. But if maturation is triggered by size then one must take account of the fact that the instantaneous growth rate of a larva depends on many factors. For larval mosquitoes it is known to depend on the weather (particularly 
temperature), diet and intra- or inter-specific competition (Araújo et al [6], Hardstone and Andreadis [9], Silberbush et al [23], Couret et al [5], Jannat and Roitberg [16]). Intra-specific larval competition occurs in some mosquito populations (Reiskind and Lounibos [17]), and this has the potential to delay larval development. Gourley, Liu and Lou [8] showed that, if maturation is triggered by size, and if larvae grow at a rate depending explicitly on time or on densities, then one obtains a model with a variable maturation delay $\tau(t)$ which is defined as the time to maturation of the cohort that matures at time $t$. This variable delay is determined by a threshold condition and is, in fact, a state-dependent delay.

\section{Model derivation}

The starting point in Gourley, Liu and Lou [8] was the supposition that the rate of change of the length $x$ of an individual larva at time $t$ is given by an expression similar to

$$
\frac{d x}{d t}=P(I(t))
$$

so the rate of growth of an individual larva depends on the total number of larvae $I(t)$. In fact, instead of (2.1), it was assumed in [8] that $d x / d t=P(t, I(t))$ which allows the growth rate to depend explicitly on $t$ as well as on $I(t)$, thereby allowing direct dependence on time and incorporating, for example, seasonal effects. If $P(I)$ in $(2.1)$ is decreasing in $I$ then (2.1) models intra-specific competition among the larvae, because the implication is that an increase in their numbers will slow down the rate at which the individual larvae physically grow, since they will find it more difficult to find food. Let $\mu_{a}$ and $\mu_{i}$ denote respectively the per-capita mortalities for adults and larvae, and $B(A(t))$ denote the egg laying rate at time $t$, taken to be a function of the number of adults $A(t)$ at that time. In [8], based on their more general expression $d x / d t=P(t, I(t))$, delay differential equations are derived that determine the numbers of adults $A(t)$ and larvae $I(t)$ in the population. When (2.1) becomes the starting point, those equations reduce to

$$
\begin{gathered}
\frac{d A(t)}{d t}=-\mu_{a} A(t)+P(I(t)) \frac{B(A(t-\tau(t)))}{P(I(t-\tau(t)))} e^{-\mu_{i} \tau(t)}, \\
\frac{d I(t)}{d t}=-\mu_{i} I(t)+B(A(t))-P(I(t)) \frac{B(A(t-\tau(t)))}{P(I(t-\tau(t)))} e^{-\mu_{i} \tau(t)},
\end{gathered}
$$

where the delay $\tau(t)$ is the maturation time for the cohort that matures at time $t$ and is determined from the threshold condition

$$
\int_{t-\tau(t)}^{t} P(I(\xi)) d \xi=l
$$

with $l$ a fixed positive constant. Maturation is triggered when a larva reaches length $l$. Smith [24] has previously considered scenarios in which individuals mature on reaching a fixed size or mass, but he took $d x / d t$ to be a function of his variable for the total number of adults, rather than larvae as in (2.1). This might be appropriate in some ecological scenarios, and it facilitates the formulation of a model consisting of just one equation determining the 
number of adults $A(t)$, rather than the coupled system (2.2)-(2.3) that determines $A(t)$ and $I(t)$ together. However, in some species larvae and adults inhabit completely different habitats (think of mosquitoes, for example). For such species it is our view that competition hampering the growth of individual larvae is likely to come mainly from other larvae, so that (2.1) becomes an appropriate starting point.

Equation (2.3) can be replaced by the integral equation

$$
I(t)=\int_{t-\tau(t)}^{t} B(A(\xi)) e^{-\mu_{i}(t-\xi)} d \xi
$$

with $\tau(t)$ again given by (2.4).

In Section 3.2 of this paper, we take

$$
P(I)=\left\{\begin{array}{cc}
P_{0}, & I \leq I_{c} \\
0, & I>I_{c}
\end{array}\right.
$$

with $P_{0}$ constant. Thus, larvae grow at a constant rate while their numbers remain below $I_{c}$, but larval development pauses completely if the threshold $I_{c}$ is exceeded. As a consequence, if larval numbers are always below $I_{c}$ then all larvae take a time $l / P_{0}$ to mature. Any pauses can only increase this time, so we always have the estimate $\tau(t) \geq l / P_{0}$. If $I(t)$ has been above $I_{c}$, falls back below $I_{c}$ and for whatever reason remains below $I_{c}$ for all subsequent time, then the model eventually assumes a constant delay format. These observations help us to prove our results on the model dynamics.

Equation (2.4) defines $\tau(t)$ uniquely if the function $P(\cdot)$ is strictly positive. This is not the case for (2.6). For that choice of $P(\cdot), P(I(t))$ is a step function of $t$ and, for most values of $t, \tau(t)$ is uniquely determined. However, for a given $t$ the function

$$
s \rightarrow \int_{t-s}^{t} P(I(\xi)) d \xi
$$

though increasing in $s$, is not necessarily strictly increasing due to the possibility of intervals of $t$ for which $I>I_{c}$. One easily appreciates the possibility that this function could equal $l$ for a whole interval of values of $s$. Because of this, for situations in which $P(\cdot)$ is non-negative, but not strictly positive everywhere, we must define $\tau(t)$ by

$$
\tau(t)=\inf \left\{s>0: \quad \int_{t-s}^{t} P(I(\xi)) d \xi=l\right\}
$$

which corresponds to $(2.4)$ if $P(\cdot)$ is strictly positive. Moreover, the set defined above is closed, so $\tau(t)$ belongs to it.

In [8], progress was made by introducing a new size-like independent variable that, for larvae, corresponds to actual physical size as measured by length. If there is a fixed threshold value for the size of a larva that triggers maturation then a transformation to a size-like independent variable generates a system with a fixed, rather than variable, time delay. This approach is useful if it can be assured that the size-like independent variable does indeed grow, strictly monotonically, as $t$ grows. The approach breaks down in this paper because we are interested in situations in which growth of larvae may pause completely, resuming at 
a later time. In this situation as a larva matures its physical size may not be in one to one correspondence with real time. Thus, in this paper we have to consider system (2.2)-(2.4) with its variable time delay $\tau(t)$. In Section 3.2 we consider the case in which the function $P(\cdot)$ in $(2.1)$ is given by $(2.6)$, so that larvae completely cease to grow if there are too many of them.

\section{Model analysis}

\subsection{Basic properties}

We first prove the following result which establishes, for the case when $P(I)$ is given by (2.6), that even at times when $P(I(t))$ is zero the quantity $P(I(t-\tau(t)))$ in the denominators of (2.2) and (2.3) is never zero. Therefore, system (2.2)-(2.4) never becomes singular.

Proposition 3.1 If $P(I)$ is given by (2.6), there exists no value of $t$ for which $P(I(t-$ $\tau(t)))=0$.

Proof. Suppose there exists $t^{*}$ such that $P\left(I\left(t^{*}-\tau\left(t^{*}\right)\right)\right)=0$. This implies that $I\left(t^{*}-\right.$ $\left.\tau\left(t^{*}\right)\right)>I_{c}$. Since the intervals of $t$ for which $I(t)>I_{c}$ are open, the implication is that $I\left(t^{*}-\left(\tau\left(t^{*}\right)-\epsilon\right)\right)>I_{c}$ for sufficiently small $\epsilon$. Since the set defined in (2.7) is closed,

$$
\int_{t^{*}-\tau\left(t^{*}\right)}^{t^{*}} P(I(\xi)) d \xi=l .
$$

Changing the lower limit of integration from $t^{*}-\tau\left(t^{*}\right)$ to $t^{*}-\left(\tau\left(t^{*}\right)-\epsilon\right)$, for sufficiently small $\epsilon$, will not change the value of the integral since both the old and new lower limits belong to the same interval of values of $t$ for which $I>I_{c}$, and the integrand $P(I(\cdot))$ remains zero throughout that interval. Thus

$$
\int_{t^{*}-\left(\tau\left(t^{*}\right)-\epsilon\right)}^{t^{*}} P(I(\xi)) d \xi=l .
$$

But this implies that $\tau\left(t^{*}\right)-\epsilon$ belongs to the set defined in (2.7), even though $\tau\left(t^{*}\right)$ is the infimum of that set. This is a contradiction.

The above proposition establishes, if $P(I)$ is given by (2.6), that $P(I(t-\tau(t)))=P_{0}$ for all $t$, and therefore that the ratio $P(I(t)) / P(I(t-\tau(t)))$ is always either 0 or 1 .

\subsection{Situation with an Allee Effect}

For the case when $P(I)$ is given by (2.6) the goal of this section is to prove that, if the model has an Allee effect and other conditions hold, including that $\mu_{i}$ is sufficiently small and $\mu_{a}$ is sufficiently large, the population goes extinct if the number of larvae becomes large enough to trigger pausing of larval development. Intuitively the mechanism is as follows. If $\mu_{i}$ is very small (relative to $\mu_{a}$ ) then larvae die much more slowly than adults. If the number of larvae exceeds $I_{c}$, so that larval development pauses, then the maturation rate drops to 
zero so the number of adults begins to decay exponentially. Those adults still alive continue to lay eggs and we assume larvae have a low per-capita death rate, with the consequence that the number of larvae $I(t)$ still increases for a while. Since the adults are dying without being replaced, the egg laying rate drops and eventually the number of larvae starts to decrease. That number would approach zero, but for the fact that it must drop below the threshold $I_{c}$, which triggers larval development once more and raises the possibility that the number of adults $A(t)$ might not approach zero. Adults and larvae can (in some parameter regimes) increase their numbers once more raising the possibility that $I(t)$ might again increase through $I_{c}$, generating oscillations in the population. The reason why extinction is the outcome if $\mu_{i}$ is very small is that, after increasing through the threshold $I_{c}$, it takes a long time for the number of larvae to begin to decline and to drop back below $I_{c}$. Over such a long time, the number of adults may have dropped below the Allee effect threshold $A_{c}$ and once that happens the population is doomed, even though larval development resumes. This creates a scenario in which an Allee effect can cause a population crash even though the initial population is not below the Allee effect threshold (and may in fact be quite large).

Mathematically, our strategy for proving that extinction is the outcome for sufficiently low $\mu_{i}$ is to show that after a sufficient amount of time the model becomes one with a constant time delay. It is useful to first prove Lemma 3.2, which applies to the situation in which the function $P(\cdot)$ in $(2.1)$ is constant. In that particular case, the equation for $A(t)$ assumes the following form in which all parameters including $\tau$ are constant:

$$
\frac{d A(t)}{d t}=-\mu_{a} A(t)+e^{-\mu_{i} \tau} B(A(t-\tau)) .
$$

Equation (3.8) exhibits an Allee effect if the egg-laying rate $B(\cdot)$ satisfies the following qualitative assumptions:

$B(A)$ is non-negative and satisfies $B(0)=0$. There exist $A_{c}>0$ and $A^{*}>A_{c}$ such that $e^{-\mu_{i} \tau} B(A)<\mu_{a} A$ when $0<A<A_{c}$ with $B(A)$ monotone increasing on that interval, $e^{-\mu_{i} \tau} B(A)>\mu_{a} A$ when $A_{c}<A<A^{*}$ and $e^{-\mu_{i} \tau} B(A)<\mu_{a} A$ when $A>A^{*}$.

Both $A_{c}$ and $A^{*}$ are steady states of (3.8). The lower steady state $A_{c}$ is the minimum viable number of adults in the sense that if $A(t)$ remains below $A_{c}$ for an amount of time at least $\tau$, then the population is doomed. The function $B(A)=p A^{2} e^{-q A}$ satisfies (3.9) for suitable $p, q>0$.

Lemma 3.2 Suppose that a solution $A(t)$ of (3.8) for $t>0$, subject to a continuous initial function $A(\theta)=A_{0}(\theta), \theta \in[-\tau, 0]$, enters the interval $\left[0, A_{c}\right]$ and remains there continuously for at least $\tau$ time units, with $A(t)<A_{c}$ for at least some of this time. Then $A(t) \rightarrow 0$ as $t \rightarrow \infty$.

Proof. The interval of values of $t$ over which $A(t)$ remains in $\left[0, A_{c}\right]$ can be taken, without loss of generality, as $t \in[-\tau, 0]$. We prove that $A(t) \in\left[0, A_{c}\right]$ for all $t \geq-\tau$. For $t \in[0, \tau]$, $A(t-\tau) \in\left[0, A_{c}\right]$ so

$$
A^{\prime}(t) \leq-\mu_{a} A(t)+\mu_{a} A(t-\tau)
$$

and thus, by comparison, $A(t) \leq \bar{A}(t)$ where $\bar{A}(t)$ satisfies

$$
\bar{A}^{\prime}(t)=-\mu_{a} \bar{A}(t)+\mu_{a} \bar{A}(t-\tau) .
$$


Moreover, for $t \in[0, \tau], A(t)$ satisfies

$$
A^{\prime}(t) \leq-\mu_{a} A(t)+\mu_{a} A_{c}
$$

and therefore

$$
A(t) \leq A(0) e^{-\mu_{a} t}+A_{c}\left(1-e^{-\mu_{a} t}\right) \leq A_{c} e^{-\mu_{a} t}+A_{c}\left(1-e^{-\mu_{a} t}\right)=A_{c}
$$

for $t \in[0, \tau]$. By induction, $A(t) \in\left[0, A_{c}\right]$ for all $t \geq-\tau$. It follows that $A(t)$ remains within the interval of values for which $B(A)$ is increasing. By standard theorems on generic convergence to equilibria for monotone systems (Smith [25]), $A(t)$ approaches some limit as $t \rightarrow \infty$ and clearly this limit must be either $A_{c}$ or zero. Suppose, for a contradiction, that $A(t) \rightarrow A_{c}$ as $t \rightarrow \infty$. Now, $A(t) \leq \bar{A}(t)$, with $\bar{A}(t)$ defined as above, and both functions have limits (this will be confirmed later in the case of $\bar{A}$ ). Thus

$$
A_{c} \leq \lim _{t \rightarrow \infty} \bar{A}(t) .
$$

We now find $\lim _{t \rightarrow \infty} \bar{A}(t)$, showing that the result contradicts (3.11). Applying the Laplace transform to (3.10), with $\mathcal{L}$ denoting the Laplace transform operator and $s$ the transform variable, gives

$$
\begin{aligned}
s \mathcal{L}\{\bar{A}(t)\}-\bar{A}(0) & =-\mu_{a} \mathcal{L}\{\bar{A}(t)\}+\mu_{a} \int_{0}^{\infty} \bar{A}(t-\tau) e^{-s t} d t \\
& =-\mu_{a} \mathcal{L}\{\bar{A}(t)\}+\mu_{a}\left[\int_{-\tau}^{0} \bar{A}(\xi) e^{-s(\tau+\xi)} d \xi+e^{-s \tau} \mathcal{L}\{\bar{A}(t)\}\right]
\end{aligned}
$$

after a substitution. Hence

$$
\mathcal{L}\{\bar{A}(t)\}=\frac{\bar{A}(0)+\mu_{a} \int_{-\tau}^{0} \bar{A}(\xi) e^{-s(\tau+\xi)} d \xi}{s+\mu_{a}-\mu_{a} e^{-s \tau}}
$$

and therefore

$$
\bar{A}(t)=\frac{1}{2 \pi i} \int_{\sigma-i \infty}^{\sigma+i \infty} e^{s t}\left[\frac{\bar{A}(0)+\mu_{a} \int_{-\tau}^{0} \bar{A}(\xi) e^{-s(\tau+\xi)} d \xi}{s+\mu_{a}-\mu_{a} e^{-s \tau}}\right] d s
$$

where the integral is the standard Bromwich integral with the quantity $\sigma$, in the limits, taken as any real number which strictly exceeds the supremum of the real parts of the zeros of the denominator in the integrand. Those zeros consist of $s=0$ and infinitely many pairs of complex conjugate zeros all satisfying $\operatorname{Re} s<0$. The existence of a complex conjugate pair of zeros with $\operatorname{Re} s \geq 0$ quickly yields a contradiction because such a pair would satisfy $\left|s+\mu_{a}\right|=\mu_{a}\left|e^{-s \tau}\right| \leq \mu_{a}\left|e^{-(\operatorname{Re} s) \tau}\right| \leq \mu_{a}$, which contradicts $\operatorname{Re} s \geq 0$ unless $s=0$. Thus, in (3.12), any real $\sigma>0$ suffices. Since all the zeroes of the denominator of the integrand of (3.12) have negative real parts, except for $s=0$, the residues of the poles all decay to zero as $t \rightarrow \infty$ except for the residue at the pole $s=0$. Keeping only the term attributable to the residue at that pole, Cauchy's residue theorem gives

$$
\lim _{t \rightarrow \infty} \bar{A}(t)=\operatorname{Res}\left[\frac{e^{s t}\left[\bar{A}(0)+\mu_{a} \int_{-\tau}^{0} \bar{A}(\xi) e^{-s(\tau+\xi)} d \xi\right]}{s+\mu_{a}-\mu_{a} e^{-s \tau}}, s=0\right]
$$


so that

$$
\lim _{t \rightarrow \infty} \bar{A}(t)=\frac{\bar{A}(0)+\mu_{a} \int_{-\tau}^{0} \bar{A}(\xi) d \xi}{1+\tau \mu_{a}}
$$

by a standard formula for calculating the residue at a simple pole. By hypothesis, $A(t)<A_{c}$ for some $t \in[-\tau, 0]$ and thus, by continuity, $A(t)<A_{c}$ on an interval of values of $t$ within $[-\tau, 0]$. Hence, from (3.11) and (3.13),

$$
A_{c} \leq \frac{\bar{A}(0)+\mu_{a} \int_{-\tau}^{0} \bar{A}(\xi) d \xi}{1+\tau \mu_{a}}<\frac{A_{c}+\tau \mu_{a} A_{c}}{1+\tau \mu_{a}}=A_{c} .
$$

This is a contradiction to the assumption that $A(t) \rightarrow A_{c}$ as $t \rightarrow \infty$. Thus, in fact, $A(t) \rightarrow 0$ and the proof is complete.

Our main result on extinction of the population where there is an Allee effect, $\mu_{i}$ is sufficiently small and $\mu_{a}$ sufficiently large, is Theorem 3.3. The quantities $c_{1}$ and $t_{c}$ referred to in the statement of the theorem are defined by

$$
c_{1}=\ln \left(\frac{I_{c}+\int_{0}^{\infty} B\left(A\left(t_{1}\right) e^{-\mu_{a} \xi}\right) d \xi}{I_{c}}\right), \quad t_{c}=t_{1}+\frac{1}{\mu_{a}} \ln \frac{A\left(t_{1}\right)}{A_{c}} .
$$

In the case when the population is driven to extinction (see Theorem 3.3 and Fig. 1), $t_{c}$ is the time at which the adult population $A(t)$ drops below the Allee effect threshold $A_{c}$. Note that $c_{1}$ decreases as the threshold $I_{c}$ increases. Therefore, as $I_{c}$ increases it becomes more difficult for condition (3.16) of Theorem 3.3 to be satisfied because the smallness assumption on $\mu_{i}$ becomes more severe. This makes sense because if $I_{c}$ is large it is less likely that $I(t)$ would exceed $I_{c}$, and therefore less likely that larval development pauses.

Theorem 3.3 Suppose that $P(I)$ is given by (2.6) and that the egg laying rate $B(A)$ satisfies (3.9). In addition, assume that $B(A)$ is differentiable with $B^{\prime}(A)$ increasing on $\left[0, A_{c}\right]$ and that $B(A)$ is bounded, with $B^{\text {sup }}=\sup _{A \in[0, \infty)} B(A)$. Suppose that at time $t_{1}$ the number of larvae $I(t)$ increases through the threshold $I_{c}$ that triggers pausing of larval development, and that $I(t)<I_{c}$ for all $t<t_{1}$. Suppose that $\mu_{a}$ is large enough so that

$$
A\left(t_{1}\right) \exp \left(-\mu_{a}\left(\frac{l}{P_{0}}+\frac{c_{1}}{\mu_{i}}\right)\right)+B^{\mathrm{sup}} e^{-\mu_{i} l / P_{0}}\left(\frac{1-e^{-\mu_{a} l / P_{0}}}{\mu_{a}}\right)<A_{c}
$$

that $\mu_{i}$ is sufficiently small, including the requirement that

$$
t_{1}+\frac{c_{1}}{\mu_{i}}>t_{c}+\frac{l}{P_{0}}
$$

and that

$$
I_{c}>B\left(A_{c}\right) \frac{l}{P_{0}} \quad \text { and } \quad \frac{l}{P_{0}} B^{\prime}\left(A_{c}\right)<1 .
$$

Then the solution of system (2.2)-(2.3) satisfies $(A(t), I(t)) \rightarrow(0,0)$ as $t \rightarrow \infty$. 
Proof. By hypothesis $I(t)$ increases through $I_{c}$, for the first time, at time $t_{1}$. Once that happens $P(I(t))=0$ for as long as $I$ remains above $I_{c}$. While that is the case, the governing equations for $A(t)$ and $I(t)$ assume the much simpler form

$$
\frac{d A(t)}{d t}=-\mu_{a} A(t), \quad \frac{d I(t)}{d t}=-\mu_{i} I(t)+B(A(t)) \quad \text { while } I>I_{c},
$$

and so, for sufficiently small $\mu_{i}, I(t)$ continues to increase for a while. The variable $A(t)$ tries to decay exponentially to zero, that would imply that $I(t)$ also eventually decreases and tries to tend to zero. However, this implies the existence of a time $t_{2}>t_{1}$ at which $I(t)$ decreases back through $I_{c}$ which, of course, changes the situation. On the interval $\left(t_{1}, t_{2}\right)$, $A(t)$ decreases exponentially. Let $t_{c}$ be the time at which $A(t)$ decreases through the Allee effect threshold $A_{c}$. We claim that $t_{2}>t_{c}+l / P_{0}$. Since $A(t)$ decreases right up to time $t_{2}$, this has the consequence that $A(t)$ has been below $A_{c}$ for an amount of time at least $l / P_{0}$. This, ultimately, will facilitate the application of Lemma 3.2 (with $\tau=l / P_{0}$ ) once we satisfy ourselves that $A(t)$ evolves according to an equation of the form (3.8) for all $t$ above some particular value. That is the strategy of this proof. To show that $t_{2}>t_{c}+l / P_{0}$ we proceed as follows. For $t \in\left(t_{1}, t_{2}\right)$ the simpler equations (3.18) apply and therefore

$$
A(t)=A\left(t_{1}\right) e^{-\mu_{a}\left(t-t_{1}\right)} .
$$

The time $t_{c}$ at which $A=A_{c}$ is therefore given by

$$
t_{c}=t_{1}+\frac{1}{\mu_{a}} \ln \frac{A\left(t_{1}\right)}{A_{c}} .
$$

The time $t_{2}$ at which $I(t)$ decreases back below the threshold $I_{c}$ can be found by integrating the second equation of (3.18) over the interval $\left(t_{1}, t_{2}\right)$, and using that $I\left(t_{1}\right)=I\left(t_{2}\right)=I_{c}$, obtaining

$$
I_{c}\left(1-e^{-\mu_{i}\left(t_{2}-t_{1}\right)}\right)=\int_{t_{1}}^{t_{2}} e^{-\mu_{i}\left(t_{2}-s\right)} B\left(A\left(t_{1}\right) e^{-\mu_{a}\left(s-t_{1}\right)}\right) d s .
$$

To show that the root $t_{2}>t_{1}$ of this equation satisfies $t_{2}>t_{c}+l / P_{0}$, let $x=t_{2}-t_{1}$. Then $x$ satisfies

$$
I_{c}\left(1-e^{-\mu_{i} x}\right)=\int_{0}^{x} e^{-\mu_{i}(x-\xi)} B\left(A\left(t_{1}\right) e^{-\mu_{a} \xi}\right) d \xi .
$$

Since $t_{2}$ is expected to be a long time beyond $t_{1}$ for small $\mu_{i}$ (due to low larval mortality), we seek a non-zero root $x$ of this equation of the form

$$
x=\frac{c_{1}}{\mu_{i}}+c_{2}+c_{3} \mu_{i}+\cdots .
$$

Substituting this into (3.19) and applying standard techniques of perturbation theory, with $\mu_{i}$ a small parameter and replacing the (large) upper limit on the integral by infinity, comparing terms independent of $\mu_{i}$ yields that

$$
I_{c}\left(1-e^{-c_{1}}\right)=e^{-c_{1}} \int_{0}^{\infty} B\left(A\left(t_{1}\right) e^{-\mu_{a} \xi}\right) d \xi .
$$


This can, of course, be solved explicitly for $c_{1}$, giving the expression in (3.14). We now have $t_{2}-t_{1}=x \approx c_{1} / \mu_{i}$ and therefore the desired inequality $t_{2}>t_{c}+l / P_{0}$ follows from the fact that $\mu_{i}$ is, by hypothesis, small enough so that (3.16) holds. There are further smallness assumptions on $\mu_{i}$ which will become apparent later.

The proof proceeds by considering separately two scenarios for $t>t_{2}$ :

CASE 1: $I(t)$ remains below $I_{c}$ for all $t>t_{2}$. In this case there is no further pausing of larval growth after time $t_{2}$. Subsequent to that time, larval development always proceeds at a steady rate, each larva taking time $l / P_{0}$ to complete its development and therefore, for all times $t>t_{2}+l / P_{0}, \tau(t)=l / P_{0}, I(t)<I_{c}$ and $I(t-\tau(t))=I\left(t-l / P_{0}\right)<I_{c}$ and therefore, using (2.6), equation (2.2) becomes

$$
\frac{d A(t)}{d t}=-\mu_{a} A(t)+B\left(A\left(t-l / P_{0}\right)\right) e^{-\mu_{i} l / P_{0}} .
$$

This equation holds only for $t>t_{2}+l / P_{0}$. To apply Lemma 3.2 (with $\tau=l / P_{0}$ ) and conclude from it that $A(t) \rightarrow 0$ as $t \rightarrow \infty$, it is sufficient to ensure that $A(t)<A_{c}$ for all $t \in\left(t_{2}, t_{2}+l / P_{0}\right)$. Our previous analysis only establishes that $A(t)$ has decreased below $A_{c}$ on a time interval prior to time $t_{2}$. Therefore we must now show that, on the interval $\left(t_{2}, t_{2}+l / P_{0}\right)$, during which $A(t)$ is likely to increase (since larval development has resumed and the maturation rate is no longer zero), $A(t)$ nevertheless rises no more than $A_{c}$. On the interval $t \in\left(t_{2}, t_{2}+l / P_{0}\right), \tau(t) \geq l / P_{0}$ and therefore, from (2.2),

$$
\frac{d A(t)}{d t} \leq-\mu_{a} A(t)+B^{\mathrm{sup}} e^{-\mu_{i} l / P_{0}} .
$$

Therefore, for $t \in\left(t_{2}, t_{2}+l / P_{0}\right)$,

$$
A(t) \leq e^{-\mu_{a}\left(t-t_{2}\right)} A\left(t_{2}\right)+B^{\mathrm{sup}} e^{-\mu_{i} l / P_{0}}\left(\frac{1-e^{-\mu_{a}\left(t-t_{2}\right)}}{\mu_{a}}\right) .
$$

But $A\left(t_{2}\right)=A\left(t_{1}\right) e^{-\mu_{a}\left(t_{2}-t_{1}\right)}=A\left(t_{1}\right) e^{-\mu_{a} c_{1} / \mu_{i}}$. Thus

$$
A\left(t_{2}+l / P_{0}\right) \leq A\left(t_{1}\right) \exp \left(-\mu_{a}\left(\frac{l}{P_{0}}+\frac{c_{1}}{\mu_{i}}\right)\right)+B^{\sup } e^{-\mu_{i} l / P_{0}}\left(\frac{1-e^{-\mu_{a} l / P_{0}}}{\mu_{a}}\right)<A_{c}
$$

by the hypothesis that $\mu_{a}$ is large enough so that (3.15) holds. We now have that $A(t)<A_{c}$ for all $t \in\left(t_{2}, t_{2}+l / P_{0}\right)$, an interval of time of length $l / P_{0}$ ending at time $t_{2}+l / P_{0}$. Since (3.20) holds after that time, it follows from Lemma 3.2 (with $\tau=l / P_{0}$ ) that $A(t) \rightarrow 0$ as $t \rightarrow \infty$. Then, equation (2.5) (recalling that $\tau(t)=l / P_{0}$ for $t$ sufficiently large) yields that $I(t) \rightarrow 0$ also.

CASE 2: subsequent to time $t_{2}$ there exists a time $t_{3}$ at which $I(t)$ once again increases through the threshold $I_{c}$. We show that this cannot, in fact, happen. From the analysis presented for case 1 , we still have that $A(t)<A_{c}$ for all $t \in\left(t_{2}, t_{2}+l / P_{0}\right)$. If $t_{3}$ is later than this we have that, for as long as $I$ remains below $I_{c}$ (which is the case up to time $t_{3}$ ), $A(t)$ satisfies (3.20) and therefore $A(t)$ still remains below $A_{c}$. There are two sub-cases to consider. 
Sub-case 2a: $t_{3}>t_{2}+l / P_{0}$. In this case $\tau\left(t_{3}\right)=l / P_{0}$ and therefore, from (2.5),

$$
I_{c}=I\left(t_{3}\right)=\int_{t_{3}-l / P_{0}}^{t_{3}} B(A(\xi)) e^{-\mu_{i}\left(t_{3}-\xi\right)} d \xi \leq B\left(A_{c}\right) \frac{l}{P_{0}}
$$

since we assume in (3.9) that $B(A)$ is increasing on $\left[0, A_{c}\right]$. The above estimate contradicts (3.17). Thus there is no $t_{3}>t_{2}+l / P_{0}$.

Sub-case 2b: $t_{3} \in\left(t_{2}, t_{2}+l / P_{0}\right)$. This possibility implies that, after $I(t)$ drops below $I_{c}$ at time $t_{2}$, the rise of $I(t)$ back to $I_{c}$ at time $t_{3}$ is relatively rapid, an indication that $I(t)$ has a relatively high second derivative on $\left(t_{2}, t_{3}\right)$. Larvae that mature just after time $t_{2}$ are larvae that were ready to mature just before the pause, which began at time $t_{1}$, and therefore the maturation time for those larvae is $\tau(t)=l / P_{0}+t_{2}-t_{1}$. The maturation time $\tau(t)$ suddenly resets itself to $l / P_{0}$ at time $t_{2}+l / P_{0}$ but we are now considering the situation when $t_{3}$ is before this time, so $\tau(t)=l / P_{0}+t_{2}-t_{1}$ for all $t \in\left(t_{2}, t_{3}\right)$. We remind the reader that the correctness of these comments about the behaviour of $\tau(t)$ is only assured where the variable $I(t)$ started below $I_{c}$ and has been above $I_{c}$ only over a single time interval $\left(t_{1}, t_{2}\right)$. Note that, for $t \in\left(t_{2}, t_{3}\right)$,

$$
t-\tau(t)<t_{3}-\left(l / P_{0}+t_{2}-t_{1}\right)<t_{2}+l / P_{0}-\left(l / P_{0}+t_{2}-t_{1}\right)=t_{1}
$$

and we are viewing $t_{1}$ as the initial time, so that $A(t-\tau(t))$ is effectively initial data. To make this more clear, we denote $A(t)=A_{0}(t)$ for times $t<t_{1}$ and view $A_{0}(\cdot)$ as given. For $t \in\left(t_{2}, t_{3}\right), I(t)<I_{c}, P(I(t))=P_{0}$ and equations (2.2) and (2.3) become

$$
\frac{d A(t)}{d t}=-\mu_{a} A(t)+M_{0}(t), \quad \frac{d I(t)}{d t}=-\mu_{i} I(t)+B(A(t))-M_{0}(t)
$$

where

$$
M_{0}(t)=B\left(A_{0}\left(t-\left(l / P_{0}+t_{2}-t_{1}\right)\right)\right) e^{-\mu_{i}\left(l / P_{0}+t_{2}-t_{1}\right)} .
$$

Note also that we have $A(t)<A_{c}$ on $\left(t_{2}, t_{3}\right)$. From (3.21),

$$
\begin{aligned}
\frac{d^{2} I(t)}{d t^{2}} & =-\mu_{i} \frac{d I(t)}{d t}+B^{\prime}(A(t))\left[-\mu_{a} A(t)+M_{0}(t)\right]-M_{0}^{\prime}(t) \\
& \leq-\mu_{i} \frac{d I(t)}{d t}+B^{\prime}(A(t)) M_{0}(t)-M_{0}^{\prime}(t)
\end{aligned}
$$

on $\left(t_{2}, t_{3}\right)$, using that $A(t)<A_{c}$ and that $B^{\prime}(A)>0$ on $\left[0, A_{c}\right]$ (by $\left.(3.9)\right)$. Now

$$
I\left(t_{3}\right)=I\left(t_{2}\right)+\left(t_{3}-t_{2}\right) I^{\prime}\left(t_{2}\right)+\int_{t_{2}}^{t_{3}}\left(t_{3}-\xi\right) I^{\prime \prime}(\xi) d \xi .
$$

Since $I\left(t_{2}\right)=I\left(t_{3}\right)=I_{c}$, using the above estimate for $I^{\prime \prime}(t)$ gives

$$
I_{c} \leq I_{c}+\left(t_{3}-t_{2}\right) I^{\prime}\left(t_{2}\right)+\int_{t_{2}}^{t_{3}}\left(t_{3}-\xi\right)\left[-\mu_{i} I^{\prime}(\xi)+B^{\prime}(A(\xi)) M_{0}(\xi)-M_{0}^{\prime}(\xi)\right] d \xi
$$


Using integration by parts on the terms involving $I^{\prime}(\xi)$ and $M_{0}^{\prime}(\xi)$,

$$
\begin{aligned}
0 \leq & \left(t_{3}-t_{2}\right) I^{\prime}\left(t_{2}\right)+\mu_{i}\left(t_{3}-t_{2}\right) I\left(t_{2}\right)-\mu_{i} \int_{t_{2}}^{t_{3}} I(\xi) d \xi \\
& +\int_{t_{2}}^{t_{3}}\left(t_{3}-\xi\right) B^{\prime}(A(\xi)) M_{0}(\xi) d \xi+\left(t_{3}-t_{2}\right) M_{0}\left(t_{2}\right)-\int_{t_{2}}^{t_{3}} M_{0}(\xi) d \xi
\end{aligned}
$$

But $I^{\prime}\left(t_{2}\right)+\mu_{i} I\left(t_{2}\right)+M_{0}\left(t_{2}\right)=B\left(A\left(t_{2}\right)\right)$. Also, neglecting the term involving the integral of $I$, but retaining the similar integral term involving $M_{0}$ even though the latter also has definite sign,

$$
0 \leq\left(t_{3}-t_{2}\right) B\left(A\left(t_{2}\right)\right)+\int_{t_{2}}^{t_{3}}\left(t_{3}-\xi\right) B^{\prime}(A(\xi)) M_{0}(\xi) d \xi-\int_{t_{2}}^{t_{3}} M_{0}(\xi) d \xi
$$

By hypothesis, $B^{\prime}(A)$ increases on $\left[0, A_{c}\right]$. Also, recall that $A\left(t_{2}\right)=A\left(t_{1}\right) e^{-\mu_{a} c_{1} / \mu_{i}}$. Therefore

$$
\begin{aligned}
\int_{t_{2}}^{t_{3}} M_{0}(\xi) d \xi & \leq\left(t_{3}-t_{2}\right) B\left(A\left(t_{1}\right) e^{-\mu_{a} c_{1} / \mu_{i}}\right)+B^{\prime}\left(A_{c}\right) \int_{t_{2}}^{t_{3}}\left(t_{3}-\xi\right) M_{0}(\xi) d \xi \\
& \leq\left(t_{3}-t_{2}\right) B\left(A\left(t_{1}\right) e^{-\mu_{a} c_{1} / \mu_{i}}\right)+\left(t_{3}-t_{2}\right) B^{\prime}\left(A_{c}\right) \int_{t_{2}}^{t_{3}} M_{0}(\xi) d \xi
\end{aligned}
$$

In the situation currently under consideration, $t_{3}-t_{2} \leq l / P_{0}$. Applying this estimate, and then rearranging,

$$
\left(1-\frac{l}{P_{0}} B^{\prime}\left(A_{c}\right)\right) \int_{t_{2}}^{t_{3}} M_{0}(\xi) d \xi \leq \frac{l}{P_{0}} B\left(A\left(t_{1}\right) e^{-\mu_{a} c_{1} / \mu_{i}}\right)
$$

and recall that $\left(l / P_{0}\right) B^{\prime}\left(A_{c}\right)<1$ (hypothesis (3.17)). Recall the definition of $M_{0}$ and note the implication that the integral in the left hand side of (3.23) only involves values of the variable $A$ prior to time $t_{1}$. We are now viewing $t_{1}$ as the initial time, and the values of the variable $A$ prior to time $t_{1}$ as given initial data, so the integral can be viewed as a given quantity depending on $\mu_{i}$ only through the factor $e^{-\mu_{i}\left(l / P_{0}+t_{2}-t_{1}\right)}$ in $(3.22)$ (but recall that $t_{2}-t_{1}$ depends on $\mu_{i}$, in fact $\left.t_{2}-t_{1} \approx c_{1} / \mu_{i}\right)$. Since this whole proof is for asymptotically small $\mu_{i}$, this exponential factor is approximately $e^{-c_{1}}$. Finally, we obtain a contradiction by letting $\mu_{i} \rightarrow 0$ in (3.23), in which limit the left hand side approaches a positive limit while the right hand side approaches zero, so that (3.23) is false for sufficiently small $\mu_{i}$.

With no $t_{3}$, we are back to case 1 and the proof of the theorem is complete.

The simulation results shown in Fig. 1 illustrate the theoretical result of Theorem 3.3. The rapid drop of the adult population $A(t)$, which then briefly grows before succumbing to its doom, is very consistent with the approach used to prove that theorem. For comparison, a situation in which adult and larval populations persist, with the same Allee effect birth function, a lower value for $\mu_{i}$ and the same $\mu_{a}$, is shown in Fig. 2. We noticed that lowering $\mu_{i}$ further to an extremely low value does always result in extinction. These observations are all consistent with Theorem 3.3 but suggest that the smallness requirement on $\mu_{i}$ in that theorem is rather a strong one. 

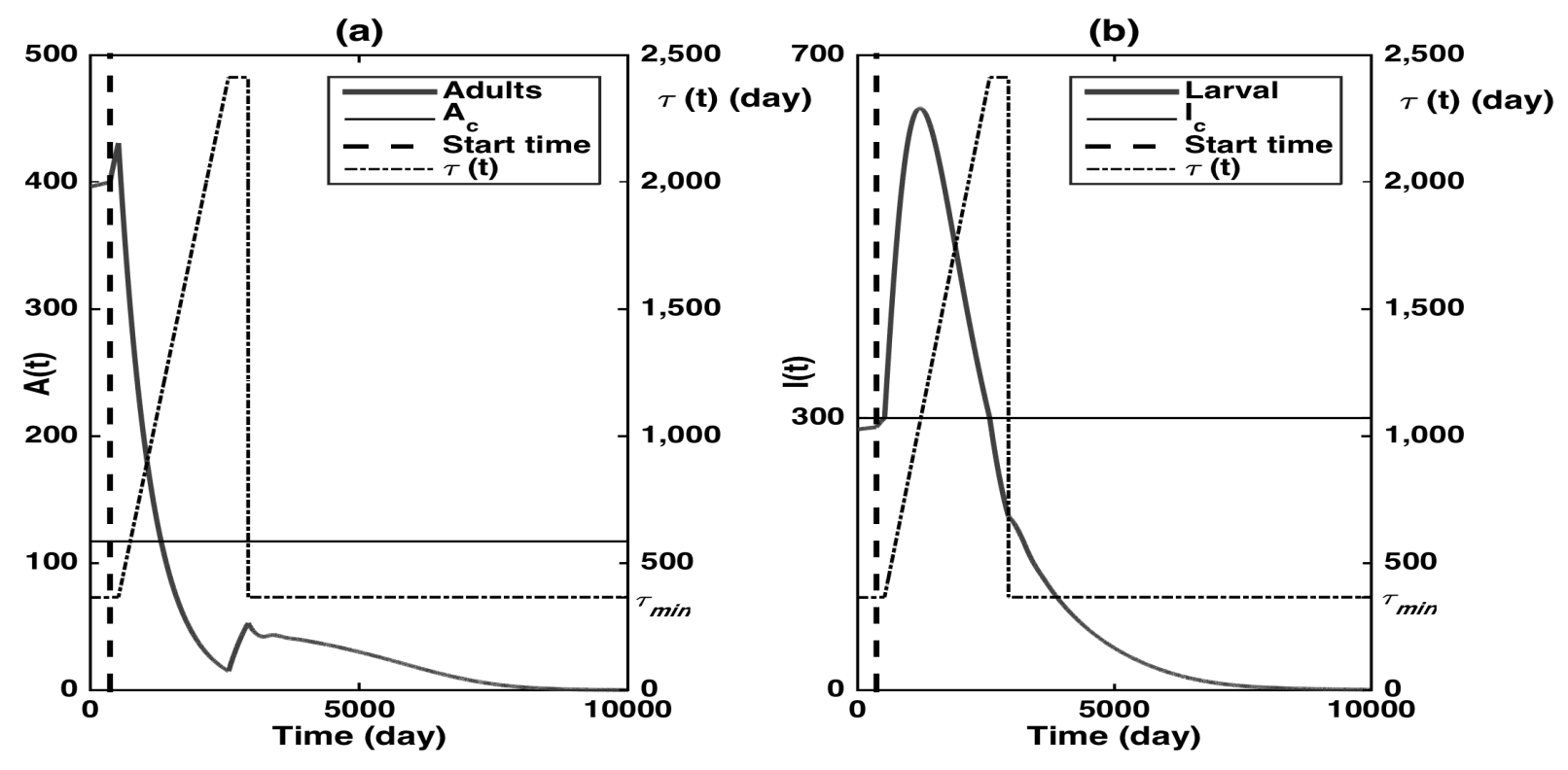

Figure 1: Simulation of (2.2)-(2.3) with $P(I)$ given by (2.6) and $B(A)=p A^{2} e^{-q A}$. Panels (a) and (b) show the adult and larval populations going extinct, even though they started large, as a consequence of the pausing of larval development causing adult numbers to drop and remain below the Allee effect threshold $A_{c}$. Note how $A(t)$ and $I(t)$ respond to changes in $\tau(t)$. Parameter values: $\mu_{a}=0.00219$ per day, $\mu_{i}=0.00082$ per day, $p=0.00005, q=0.005$, $P_{0}=0.00274$ per day, $I_{c}=300$ and $l=1$. For each panel the left vertical axis relates to $A(t)$ or $I(t)$, the right one relates to $\tau(t)$, and $\tau_{\min }=l / P_{0}$. 

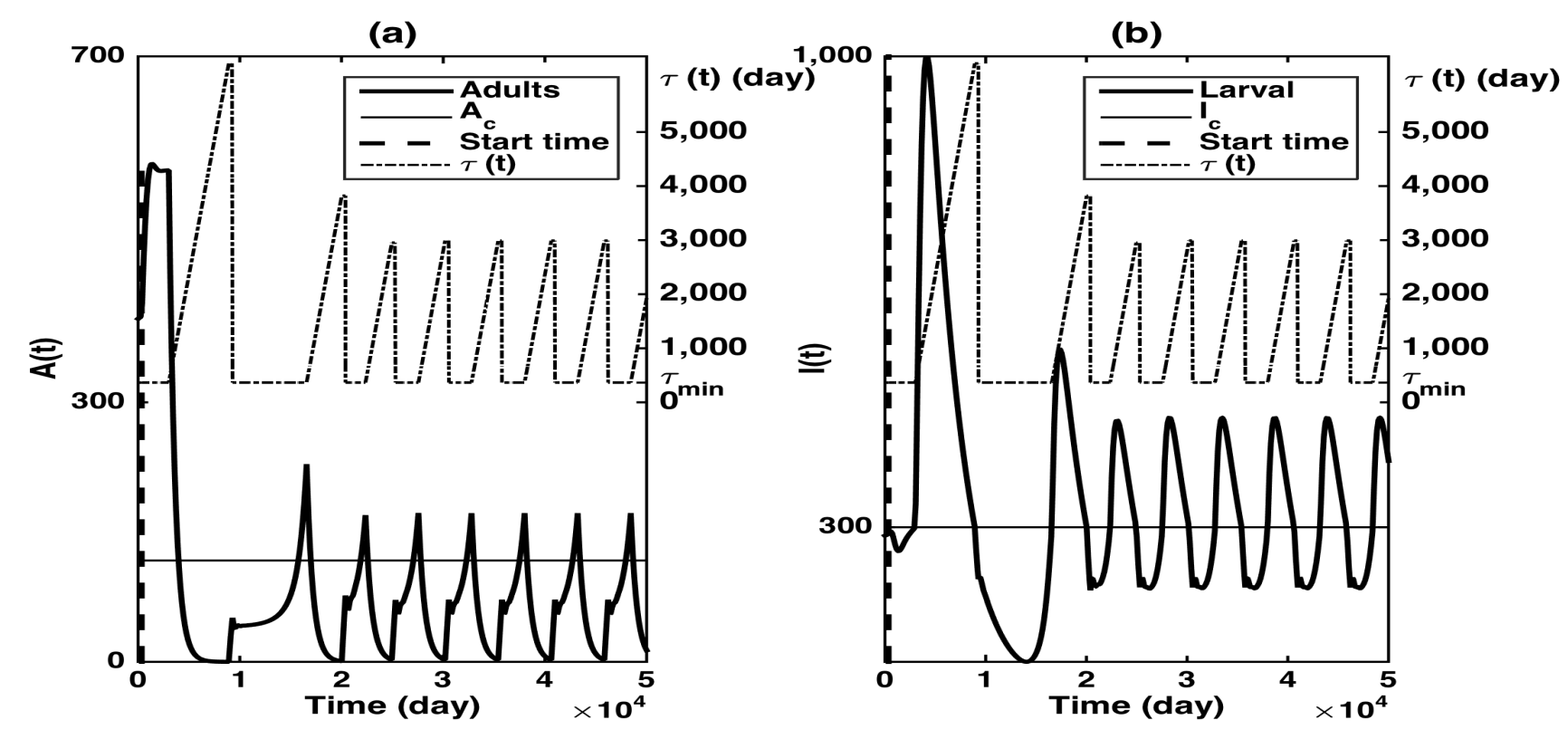

Figure 2: Simulation of (2.2)-(2.3) with $P(I)$ given by $(2.6)$ and $B(A)=p A^{2} e^{-q A}$. Here, adult numbers do drop below the Allee effect threshold $A_{c}$ but never stay below it for long, and a periodic solution results. Parameter values: $\mu_{a}=0.00219$ per day, $\mu_{i}=0.00027$ per day, $p=0.00005, q=0.005, P_{0}=0.00274$ per day, $I_{c}=300$ and $l=1$. For each panel the left vertical axis relates to $A(t)$ or $I(t)$, the right one relates to $\tau(t)$, and $\tau_{\min }=l / P_{0}$.

\subsection{Oscillatory solutions}

The last section presented the novel finding that pausing of larval development can, in some circumstances, cause a population to go extinct. In this section we turn our attention to another issue, that such pauses can give rise to oscillatory solutions where such solutions would otherwise not exist. In this section we make the following assumption about the egg-laying rate $B(\cdot)$ :

$B(A)$ is a strictly increasing differentiable function with $B(0)=0$; there exists $A_{0}^{*}>0$ such that $B(A) e^{-\mu_{i} l / P_{0}}>\mu_{a} A$ when $0<A<A_{0}^{*}$, and $B(A) e^{-\mu_{i} l / P_{0}}<\mu_{a} A$ when $A>A_{0}^{*}$.

If (3.24) holds, $A_{0}^{*}$ is the $A$ component of the unique positive steady state of model (2.2)(2.3) in the case when $P(I) \equiv P_{0}$, so that $\tau(t) \equiv l / P_{0}$ (or, equivalently, $I_{c}=\infty$ in (2.6)). The reason why we wish to make this assumption, in particular having $B(A)$ increasing in $A$, has to do with the properties of the solutions of $(2.2)-(2.3)$ in situations when $\tau(t)$ is constant. In this situation the equation for the number of adults $A(t)$ reduces to (3.20). If $P(I)$ is given by (2.6), one can easily gain some insight about the likely behaviour of solutions of (2.2)-(2.3) by first imagining that the threshold $I_{c}$ is extremely large (relative to the $I$ component $I^{*}$ of the unique positive steady state - see further down.) If that is the case, $I(t)$ 
might never reach $I_{c}$, so that larval development never pauses and $\tau(t) \equiv l / P_{0}$. But then, for all but special initial conditions, the variables $A(t)$ and $I(t)$ will simply approach their respective steady state values because that is the generic solution behaviour in the situation when $B(A)$ is increasing (Smith [25]). The assumption that $B(A)$ is increasing therefore assures us that any oscillatory behaviour we find in the solutions of (2.2)-(2.3) is a direct consequence of pausing or slowing down of larval development as a consequence of larval competition, and not a periodic solution that would have arisen anyway. More precisely, we want to make sure that equation (3.20) does not have periodic solutions.

If $P(I)$ is given by $(2.6)$ and $B(\cdot)$ satisfies (3.24) then it is easy to see why oscillatory behaviour in solutions of (2.2)-(2.3) might arise for some parameter values. Imagine the threshold $I_{c}$ is set to an extremely large value. Then (for realistic initial data) one expects that the population will never notice the threshold or its effect on larval development, $A(t)$ evolves according to (3.20) and, generically, $(A, I)$ tends to a unique steady state that we call $\left(A_{0}^{*}, I_{0}^{*}\right)$. But imagine that we reduce $I_{c}$ to a value below $I_{0}^{*}$. Then $I_{0}^{*}$ can no longer be the $I$ component of a steady state, since the number of larvae cannot hold itself above $I_{c}$. This was explained near the beginning of Section 3.2; recall that while $I$ is above $I_{c}$ the evolution equations are (3.18). After a while, $I(t)$ must drop back below $I_{c}$ after which the maturation terms in $(2.2)-(2.3)$ become active. Moreover, $I(t)$ cannot remain below $I_{c}$ forever, because if it did then the delay $\tau(t)$ would become constant after a while, the $A$ equation would become (3.20) and, since we now assume that $B(\cdot)$ satisfies (3.24), the implication is that $(A(t), I(t))$ would try to approach $\left(A_{0}^{*}, I_{0}^{*}\right)$. However, $I(t)$ would have to rise through $I_{c}$, which would again trigger pausing of larval development and the cycle repeats. It is therefore clear that we can anticipate oscillatory (though not necessarily periodic) behaviour.

In the case of (2.6), the fact that the maturation terms in (2.2)-(2.3) suddenly vanish as $I$ increases through $I_{c}$ implies that, although the solution is continuous, the derivatives of the variables are discontinuous at the times at which $I(t)$ increases or decreases through $I_{c}$. This lack of smoothness led us to analytically investigate the existence of oscillatory solutions for smooth, decreasing but strictly positive choices of $P(I)$ rather than the choice (2.6), which we abandon for the remainder of the analysis of this section (though we still use (2.6) in the simulation shown in Fig. 3).

For the analysis related to Theorem 3.4, we assume that $P(I)$ satisfies:

$P(0)=P_{0} \in(0, \infty) ; P(I)$ is strictly decreasing, differentiable and strictly positive for all $I$.

If $P(I)$ satisfies (3.25) then, in principle, model (2.2)-(2.3) may have a positive steady state, though it is no longer automatically an attractor. When (3.25) holds, steady state components $A^{*}$ and $I^{*}$ satisfy

$$
\begin{aligned}
\mu_{a} A^{*} & =B\left(A^{*}\right) \exp \left\{-\frac{\mu_{i} l}{P\left(I^{*}\right)}\right\}, \\
\mu_{i} I^{*} & =B\left(A^{*}\right)\left(1-\exp \left\{-\frac{\mu_{i} l}{P\left(I^{*}\right)}\right\}\right) .
\end{aligned}
$$

A single equation can be obtained for $A^{*}$ :

$$
\mu_{a} A^{*}=B\left(A^{*}\right) \exp \left\{-\frac{\mu_{i} l}{P\left(\left(B\left(A^{*}\right)-\mu_{a} A^{*}\right) / \mu_{i}\right)}\right\} .
$$


In the case when $P(I) \equiv P_{0}$ we call the steady state $\left(A_{0}^{*}, I_{0}^{*}\right)$; and the quantity $A_{0}^{*}$ is also defined by assumption (3.24).

In Gourley, Liu and Lou [8], a linear stability analysis of the steady state $\left(A^{*}, I^{*}\right)$ was carried out and complex variable theory was used to obtain sufficient conditions for its local stability. The possibility of a Hopf bifurcation to periodic solutions was not considered. Here, we will demonstrate that as a certain parameter $\epsilon$ (defined below) is varied it is possible for a pair of complex conjugate eigenvalues of the linearised system to cross the imaginary axis, which is suggestive of a Hopf bifurcation. There is no way to prove directly that the characteristic equation can have purely imaginary roots and we do not even attempt a fully rigorous Hopf bifurcation analysis. However we will demonstrate, indirectly, that a pair of complex conjugate eigenvalues of the linearised system crosses the imaginary axis. We also present numerical evidence for the existence of oscillatory solutions. In [8], for a general $P(I)$, the characteristic equation of the linearisation of system (2.2)-(2.3) at the steady state $\left(A^{*}, I^{*}\right)$ was obtained. Adapting to the slightly different notation of the present paper, solutions of the linearised system proportional to $\exp (\lambda t)$ exist whenever $\Lambda:=\lambda l / P\left(I^{*}\right)$ satisfies

$$
\frac{P\left(I^{*}\right)}{l}\left(\Lambda+\hat{\mu}_{a}\right)-B^{\prime}\left(A^{*}\right) e^{-\left(\Lambda+\hat{\mu}_{i}\right)}=\frac{\epsilon B^{\prime}\left(A^{*}\right)\left(\Lambda+\hat{\mu}_{i}\right) k(\Lambda) k\left(\Lambda+\hat{\mu}_{i}\right)}{1+\epsilon k(\Lambda)}
$$

where

$$
\hat{\mu}_{i}=\frac{\mu_{i} l}{P\left(I^{*}\right)}, \quad \hat{\mu}_{a}=\frac{\mu_{a} l}{P\left(I^{*}\right)}, \quad \epsilon=\frac{l B\left(A^{*}\right) P^{\prime}\left(I^{*}\right) e^{-\hat{\mu}_{i}}}{P^{2}\left(I^{*}\right)}
$$

and

$$
k(x)=\frac{1-e^{-x}}{x} .
$$

We prove the following theorem suggesting the onset of oscillatory behaviour in certain circumstances.

Theorem 3.4 Suppose that $B(A)$ satisfies (3.24), $P(I)$ satisfies (3.25) and

$$
B^{\prime}\left(A^{*}\right) \exp \left(-\mu_{i} l / P\left(I^{*}\right)\right)<\mu_{a}<B^{\prime}\left(A^{*}\right) .
$$

Then as $\epsilon$ is varied between 0 and $-\infty$ a pair of complex conjugate roots of the characteristic equation (3.29) crosses the imaginary axis.

Proof. Any complex roots of (3.29) occur in complex conjugate pairs. First consider the situation when $\epsilon=0$. It can easily be shown (see [8]) that in this situation all the roots $\Lambda$ of (3.29) satisfy $\operatorname{Re} \Lambda<0$; here we use the left inequality in (3.32).

Now imagine that $\epsilon$ is a negative number of large magnitude. Let

$$
f(\Lambda)=\frac{P\left(I^{*}\right)}{l}\left(\Lambda+\hat{\mu}_{a}\right)-B^{\prime}\left(A^{*}\right) e^{-\left(\Lambda+\hat{\mu}_{i}\right)}
$$

and

$$
g(\Lambda)=\frac{\epsilon B^{\prime}\left(A^{*}\right)\left(\Lambda+\hat{\mu}_{i}\right) k(\Lambda) k\left(\Lambda+\hat{\mu}_{i}\right)}{1+\epsilon k(\Lambda)}
$$

and note that $f(\infty)=\infty$ and $g(\infty)=0$. Therefore, if $g(0)>f(0)$, i.e. if

$$
\frac{\epsilon B^{\prime}\left(A^{*}\right)}{1+\epsilon}\left(1-e^{-\mu_{i} l / P\left(I^{*}\right)}\right)>\mu_{a}-B^{\prime}\left(A^{*}\right) e^{-\mu_{i} l / P\left(I^{*}\right)},
$$


then the characteristic equation (3.29) has at least one real positive root. By the right hand inequality of (3.32), inequality (3.33) does hold for large magnitude negative $\epsilon$. Thus, as $\epsilon$ varies between 0 and $-\infty$ at least one root of the characteristic equation (3.29) must cross the imaginary axis. But note also that $g(0)>f(0)$ implies that $\Lambda=0$ cannot be a root of (3.29). Thus, roots must cross the imaginary axis as complex conjugate pairs.

Remark. For parameter values satisfying (3.32) note that the proof works by taking $\epsilon$ as a negative number of sufficient magnitude. Note also that $\epsilon$, defined in (3.30), is related to the slope of the function $P(I)$ at $I=I^{*}$. These remarks are very consistent with our earlier intuitive comments where we explained that, in the case when $P(I)$ is given by (2.6), as the threshold $I_{c}$ is decreased below the steady state component $I^{*}$ we expect the onset of oscillatory behaviour. In that particular step function case we might formally state that $P^{\prime}\left(I^{*}\right)=-\infty$ at the bifurcation.

Fig. 3 shows a periodic solution of (2.2)-(2.3) for the case when $P(I)$ is given by (2.6) with a monotone choice for $B(A)$. Periodicity occurs only in certain parameter regimes but is induced directly by the pausing of larval development at high densities.
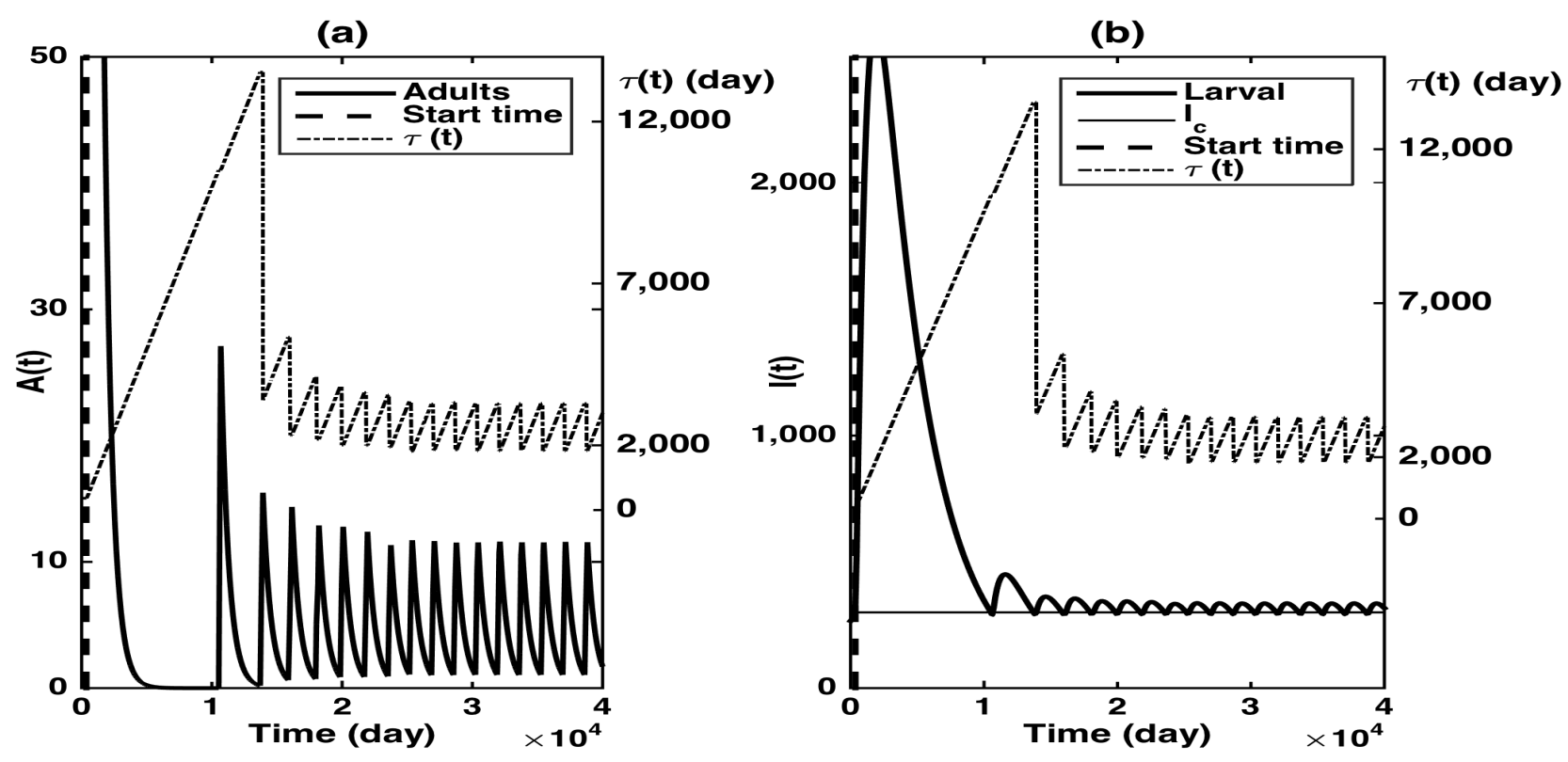

Figure 3: Simulation of (2.2)-(2.3) with $P(I)$ given by $(2.6)$ and $B(A)=p\left(1-e^{-q A}\right)$, an increasing birth function that cannot give rise to periodic solutions in situations in which $\tau(t)$ is constant. Parameter values: $\mu_{a}=0.00164$ per day, $\mu_{i}=0.00027$ per day, $p=4.10959$, $q=0.005, P_{0}=0.00274$ per day, $I_{c}=300$ and $l=1$. Note the periodicity in $A(t)$ and $I(t)$ even though $B(A)$ is monotone here. The periodicity is attributable directly to the time variation in the maturation time $\tau(t)$ caused by pausing of larval development. Note that $I(t)$ oscillates about $I_{c}$. For each panel the left vertical axis relates to $A(t)$ or $I(t)$ and the right one relates to $\tau(t)$. 


\section{Conclusion}

This paper has shown that, where diapause is induced by food deprivation because of competition among larvae, and there is an Allee effect, in some circumstances the outcome may be extinction. One way to interpret this finding is that, where such conditions prevail in nature, one would expect to see evidence for selection against diapause. What actually happens is that, in some species, the individuals may undergo premature metamorphosis if starved of food. Terao et al [27] recently studied this phenomenon in the bean blister beetle Epicauta gorhami. The larvae of this six-instar species feed only on grasshopper egg pods, which tend to be found only in low density. In laboratory experiments in which the fourth instar larvae of E. gorhami were subject to food deprivation, a tendency for premature pupation directly from the fourth instar was noted, leading to the premature emergence of a small adult. In addition to E. gorhami, premature pupation has been noted in several other insect species that use restrictive food resources. Examples include the longicorn beetle Psacothea hilaris (Shintani et al [22]) which, if it reaches the fourth instar, can pupate if it becomes starved. The situation is similar for the dung beetle Onthophagus taurus, which seem to be able to pupate if they have adequate food up to the first five days of the final instar and a weight of about $58 \%$ of mean peak weight (Shafiei et al [21]), and the beetle Dacne picta the larvae of which, if sufficiently developed, pupate around 7 days after the removal of their food resource (Sato and Suzuki [20]). The fact that premature pupation has evolved in spite of some disadvantages (for example, smaller adults may be less fecund) suggests that it evolved because of a fine balance between extinction and survival. In other words, a localised population that did not adopt the trait would have gone extinct.

Our most novel result of this paper, that diapause may case extinction in some circumstances, applies only if there is an Allee effect. Allee effects are known in various animal taxa and arise from at least six known mechanisms of which, for insects, mate limitation, cooperative defense and dispersal may be particularly relevant. Kramer et al [13] discuss the evidence of Allee effects in various taxa. They are often associated with fish, but this may be because of greater numbers of studies of fish populations due to the availability of large data sets on long term dynamics. In some taxonomic groups there is less evidence for Allee effects but, as stressed in [13], this does not suggest lower incidence in those groups. Rather, it suggests a need for more studies that collect the type of data necessary. One study of the Allee effect in insects is that of Hopper and Roush [10] who describe the role of an Allee effect in driving introduced populations extinct, whereby diffusion into a new environment may lead to densities that are low enough to result in a failure to mate.

We also noted the potential for diapause-induced periodic solutions where diapause is initiated when the number of larvae exceeds a threshold. The intuitive potential for the population to oscillate is clear, but it happens only where the threshold for triggering diapause is not too large, so that there is a possibility of larval numbers exceeding it. Otherwise, if nonlinearities impose bounds on the numbers of larvae and adults, then those numbers could simply approach equilibrium values (or exhibit more complex dynamics, staying below the bounds), diapause never actually happening. Though there is a clear reason for oscillatory behaviour in a population that experiences diapause when larval numbers are too high, we should point out that this is oscillatory behaviour in a single species autonomous population model that occurs even when the egg laying rate $B(\cdot)$ is monotone increasing. Usually in 
single species delay models periodic solutions arise as a consequence of a negative feedback attributable to the use of a non-monotone $B(\cdot)$ modelling a decrease in egg laying activity at higher densities. Competition for a single resource does not usually result in cyclic behaviour in models with low numbers of species, although it may do so where there are multiple species competing for multiple resources (Huisman and Weissing [12, 11], Sommer and Worm [26]). But, in the model of this paper, cyclic behaviour can occur even for an increasing $B(\cdot)$ and the origin of the oscillations is halting or slowing down of larval growth because of intra-specific competition among larvae. In other words we have cyclic behaviour, directly attributable to competition, in a single species model in which the individuals compete for just one resource. This is an important finding in its own right.

\section{Acknowledgements.}

The research of HB was supported by the Hong Kong Research Grants Council (GRF Grant HKBU 200113). SG's work was supported by the Leverhulme Trust, under Research Fellowship No. RF-2014-512. SG also thanks Hong Kong Baptist University and the University of Wyoming for their support. YX was supported by the UC LEAF and Simons Foundation (Collaboration Grants, No. 429551). We thank the referees for their comments.

\section{References}

[1] H.G. ANDREWARTHA, Diapause in relation to the ecology of insects, Biol. Rev., 27 (1952), pp. 50-107.

[2] N. BLAKLEY, Life history significance of size-triggered metamorphosis in milkweed bugs (Oncopeltus), Ecology, 62 (1981), pp. 57-64.

[3] V. CALLIER and H.F. NIJHOUT, Control of body size by oxygen supply reveals sizedependent and size-independent mechanisms of molting and metamorphosis, PNAS, 108 (2011), pp. 14664-14669.

[4] C. COMBES, Parasitism: The Ecology and Evolution of Intimate Interactions, University of Chicago Press, 2001.

[5] J. COURET, E. DOTSON and M.Q. BENEDICT, Temperature, larval diet, and density effects on development rate and survival of Aedes aegypti (Diptera: Culicidae), PLoS One, 9 (2014), e87468.

[6] M. da-SILVA ARAÚJO, L.H.S. GIL and A. de-ALMEIDA e-SILVA, Larval food quantity affects development time, survival and adult biological traits that influence the vectorial capacity of Anopheles darlingi under laboratory conditions, Malaria J., 11 (2012), 261.

[7] F. GONZÁLEZ-CANDELAS, J.L. MÉNSUA \& A. MOVA, Larval competition in Drosophila melanogaster: effects on development time, Genetica, 82 (1990), pp. 3344. 
[8] S.A. GOURLEY, R. LIU and Y. LOU, Intra-specific competition and insect larval development: a model with time-dependent delay, Proc. Roy. Soc. Edinburgh, Sect A. (to appear).

[9] M.C. HARDSTONE and T.G. ANDREADIS, Weak larval competition between the invasive mosquito Aedes japonicus japonicus (Diptera: Culicidae) and three resident container-inhabiting mosquitoes in the laboratory, J. Med. Entomol., 49 (2012), pp. $277-285$.

[10] K.R. HOPPER and R.T. ROUSH, Mate finding, dispersal, number released, and the success of biological control introductions, Ecol. Entomol., 18 (1993), pp. 321-331.

[11] J. HUISMAN \& F.J. WEISSING, Biodiversity of plankton by species oscillations and chaos, Nature, 402 (1999), pp. 407-410.

[12] J. HUISMAN \& F.J. WEISSING, Oscillations and chaos generated by competition for interactively essential resources, Ecol. Res., 17 (2002), pp. 175-181.

[13] A.M. KRAMER, B. DENNIS, A.M. LIEBHOLD \& J.M. DRAKE, The evidence for Allee effects, Popul. Ecol., 51 (2009), pp. 341-354.

[14] D.J. MADDER, G.A. SURGEONER and B.V. HELSON, Induction of diapause in Culex Pipiens and Culex Restuans (Diptera: Culicidae) in southern Ontario, The Canadian Entomologist, 115 (1983), pp. 877-883.

[15] M.E. MOELlER, E.T. DANIELSEN, R. HERDER, M.B. O'CONNOR and K.F. REWITZ, Dynamic feedback circuits function as a switch for shaping a maturation-inducing steroid pulse in Drosophila, Development, 140 (2013), pp. 4730-4739.

[16] K. NOOR-E JANNAT and B.D. ROITBERG, Effects of larval density and feeding rates on larval life history traits in Anopheles gambiae s.s. (Diptera: Culicidae), J. Vector Ecology, 38 (2013), pp. 120-126.

[17] M.H. REISKIND and L.P. LOUNIBOS, Effects of intraspecific larval competition on adult longevity in the mosquitoes Aedes aegypti and Aedes albopictus, Med. Vet. Entomol., 23 (2009), pp. 62-68.

[18] K.F. REWITZ, N. YAMANAKA and M.B. O'CONNOR, Developmental checkpoints and feedback circuits time insect maturation, Curr. Top. Dev. Biol., 103 (2013), pp. $1-33$.

[19] R.A. RING, Photoperiodic control of diapause induction in the larva of Lucilia Caesar L. (Diptera: Calliphoridae), J. Exp. Biol., 46 (1967), pp. 117-122.

[20] T. SATO \& A. SUZUKI, Effect of starvation and feeding of larvae during 4th stadia on pupation and adult size in Dacne picta (Coleoptera: Erotylidae), Applied Entomology and Zoology, 36 (2001), pp. 189-197. 
[21] M. SHAFIEI, A.P. MOCZEK and H.F. NIJHOUT, Food availability controls the onset of metamorphosis in the dung beetle Onthophagus taurus (Coleoptera: Scarabaeidae), Physiol. Entomol., 26 (2001), pp. 173-180.

[22] Y. SHINTANI, F.N. MUNYIRI \& Y. ISHIKAWA, Change in significance of feeding during larval development in the yellow-spotted longicorn beetle, Psacothea hilaris, J. Insect Physiol., 49 (2003), pp. 975-981.

[23] A. SILBERBUSH, I. TSURIM, R. ROSEN, Y. MARGALITH and O. OVADIA, Speciesspecific non-physical interference competition among mosquito larvae, PLoS One, 9 (2014), e88650.

[24] H.L. SMITH, Reduction of structured population models to threshold-type delay equations and functional-differential equations: a case study, Math. Biosci., 113 (1993), pp. 1-23.

[25] H.L. SMITH, Monotone Dynamical Systems: An Introduction to The Theory of Competitive and Cooperative Systems, Mathematical Surveys and Monographs, 41, American Mathematical Society, Providence, RI, 1995.

[26] U. SOMMER \& B. WORM, Competition and Coexistence, Springer Science and Business Media, 2012.

[27] M. TERAO, Y. HIROSE and Y. SHINTANI, Food-availability dependent premature metamorphosis in the bean blister beetle Epicauta gorhami (Coleoptera: Meloidae), a hypermetamorphic insect that feeds on grasshopper eggs in the larval stage, Entomological Science, 18 (2015) pp. 85-93. 